\title{
Spontaneous external rupture of hydrocephalus after fontanelle closure: a case report and review of literature
}

\author{
Varidh Katiyar $^{1} \cdot$ Kanwaljeet Garg $^{1}$ (D) $\cdot$ Ramesh Doddamani $^{1} \cdot$ Pankaj Kumar Singh ${ }^{1} \cdot$ Manmohan Singh $^{1}$. \\ P. Sarat Chandra ${ }^{1}$
}

Received: 15 April 2020 / Accepted: 1 October 2020 / Published online: 6 October 2020

(C) Springer-Verlag GmbH Germany, part of Springer Nature 2020

\begin{abstract}
We report a 2-year-old child with congenital hydrocephalus who presented with a frontal meningocoele due to ventriculosubgaleal fistula secondary to hydrocephalus (internal rupture), which gradually ruptured spontaneously through the skin. This case is unique given its association with occipital encephalocele and is only the second case to present with external rupture beyond the age of 1 year. Further, the ventricles have ruptured through the skull, after the closure of anterior fontanelle, into the subgaleal space. It challenges the present understanding that external rupture occurs only through an open anterior fontanelle.
\end{abstract}

Keywords Spontaneous rupture $\cdot$ External rupture $\cdot$ Hydrocephalus $\cdot$ Fontanelle closure $\cdot$ Subgaleal space

\section{Background}

Hydrocephalus is one of the most common causes of neurosurgical consultation in the pediatric age group. The spectrum of cases of hydrocephalus differs widely among the populations with different socio-cultural and economic backgrounds. In developing countries with more influence of alternative medical practices and poverty resulting in unaffordability, adequate intervention is usually delayed. This results in some bizarre complications, unheard in the developed countries in present times. Few cases with spontaneous external rupture through the open

Kanwaljeet Garg

kanwaljeet84@gmail.com

Varidh Katiyar

katiyar.varidh@gmail.com

Ramesh Doddamani

drsdramesh@gmail.com

Pankaj Kumar Singh

drpankajsingh11@gmail.com

Manmohan Singh

manmohan1972@gmail.com

P. Sarat Chandra

saratpchandra@gmail.com

1 Department of Neurosurgery, All India Institute of Medical Sciences, 720, CNC, AIIMS, New Delhi 110029, India anterior fontanelle have been reported in the literature [1-4]. We report a child with congenital hydrocephalus who presented with a frontal meningocele due to ventriculo-subgaleal fistula secondary to hydrocephalus (internal rupture), which gradually ruptured spontaneously through the skin.

\section{Case}

A 2-year-old boy born at full term by normal vaginal delivery presented to our outdoor clinic with complaints of a cystic swelling in the right frontal region and occipital region since birth. Though he had no focal neurological deficits, but the developmental milestones were delayed for age in all domains. At presentation, he was able to speak only monosyllables and was able to walk with support. There was bony deformity in the right frontal region as well, with scalloping of the skull and everted bony edges around the skull defect. He was diagnosed with multiple cranial meningocoeles including one in the occipital region and frontal region. He also had a swelling in the occipital region that was soft and around $4 \times$ $4 \mathrm{~cm}$ in size. This was diagnosed as an occipital encephalocele. He also had skin erosions over the right frontal swelling and had complaints of occasional watery discharge from them (Fig. 1). While in the hospital, there was rupture at the ulcer site with CSF leak through the ulcer. There was a resultant decrease in the size of the swelling of the right frontal region. The computed tomography scan before the leak 


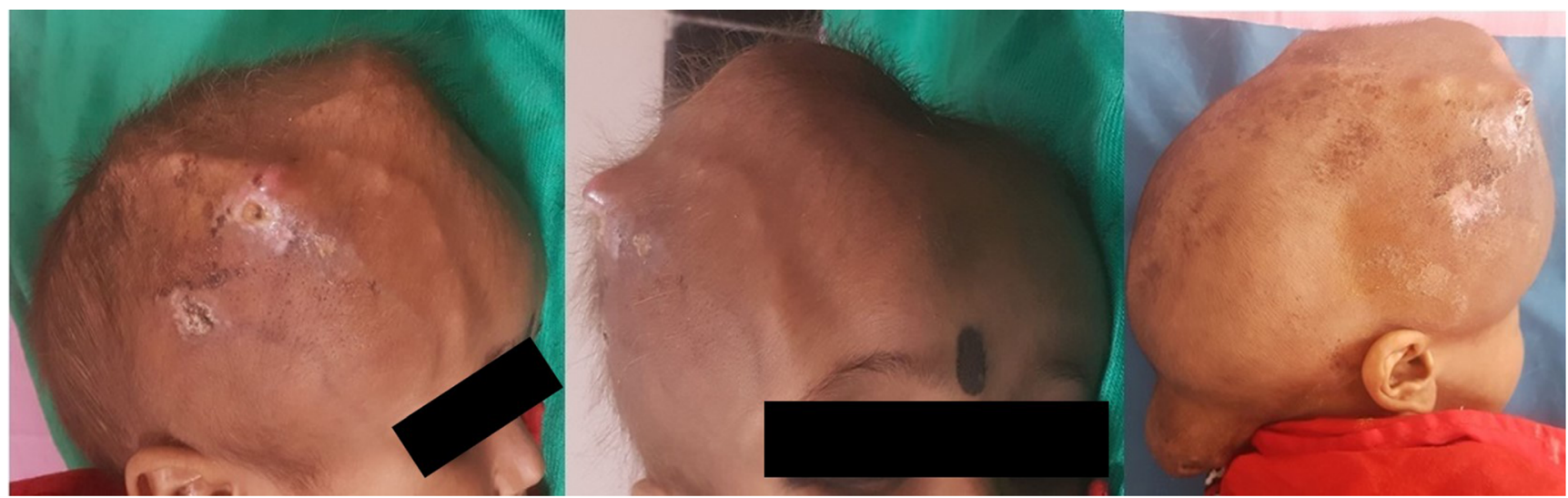

Fig. 1 Clinical picture of the boy showing the subgaleal collection of CSF with overlying skin ulceration and bony deformity

revealed enlarged lateral ventricles (right side more than the left side). The right lateral ventricle was continuous with the subgaleal swelling communicating through a defect in the right frontal bone. The outer table of the skull around the defect was also deficient over a large area with everted bony edges at the margins of the area of resorption (Fig. 2). The magnetic resonance imaging (MRI) revealed significant thinning of the cerebral cortex over the enlarged ventricles and wide area of communication between the ventricles and subgaleal collection. It also showed the presence of occipital encephalocele (Fig. 3).

The patient was planned for ventriculoperitoneal shunt followed by repair of dural defect and cranioplasty. Right occipital medium pressure Chhabra shunt, using a slit and spring valve mechanism, was placed. His postoperative course was uneventful and he was discharged after 5 days. CT scan was done on postoperative day 1 which shows adequate placement of shunt in the frontal horn (Fig. 4). The skin erosions gradually healed over 1 month. In addition, the encephalocele also reduced in size gradually. At the time of last follow-up, the patient has progressed in the developmental milestones across all domains and has shown good catch-up development; however, he is still significantly behind his chronological age. He is now able to climb stairs one at a time, speak 3 word sentences, and knows his name. Definitive surgery is planned for encephalocele but it has been delayed as elective procedures are suspended because of the COVID 19 pandemic.

\section{Discussion}

Hydrocephalus requires timely intervention to prevent the development of complications like thinning of cortical mantle

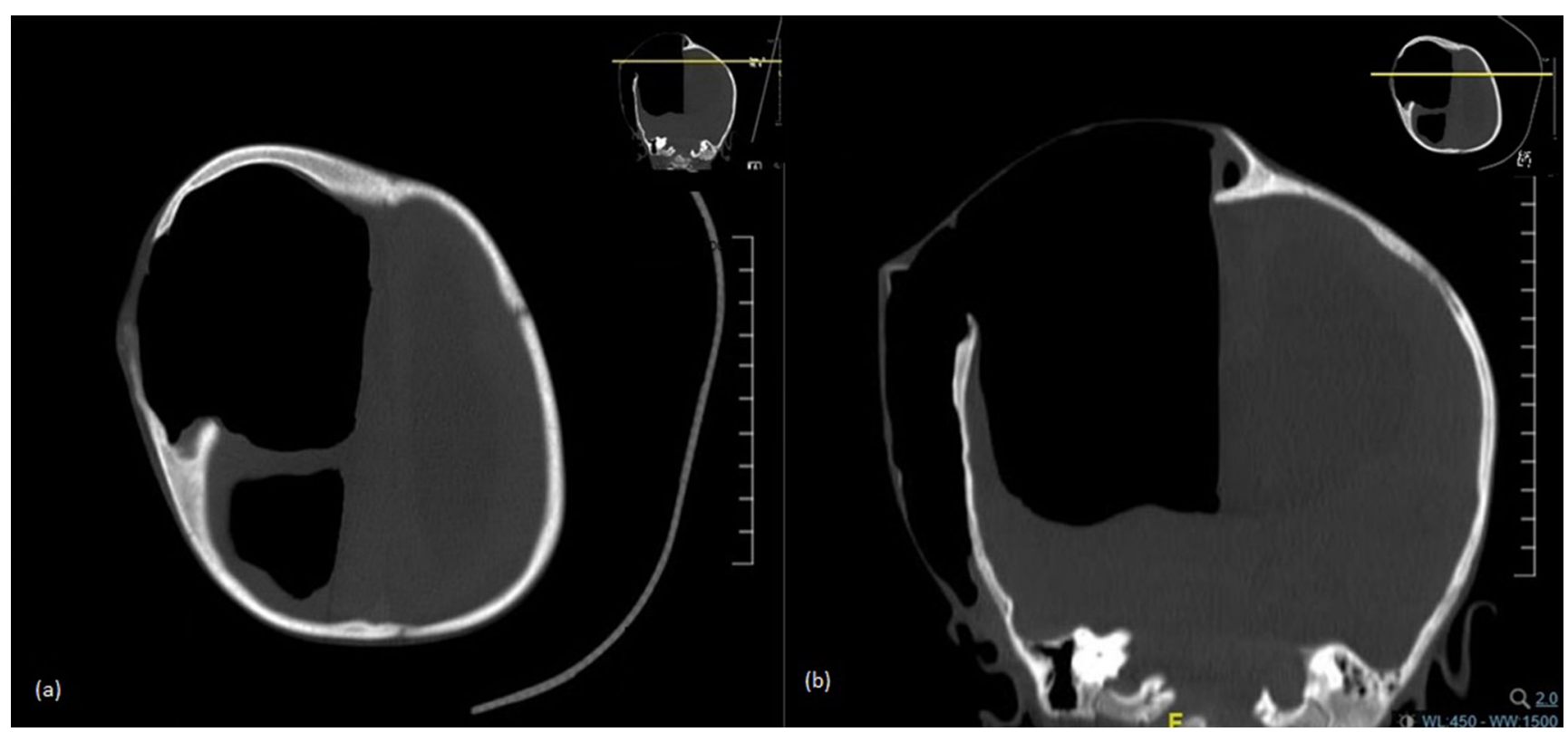

Fig. 2 Computed tomography scan showing the bony deformity and the defect through which ventricles are communicating with the subgaleal collection. a Axial section. b Coronal section 

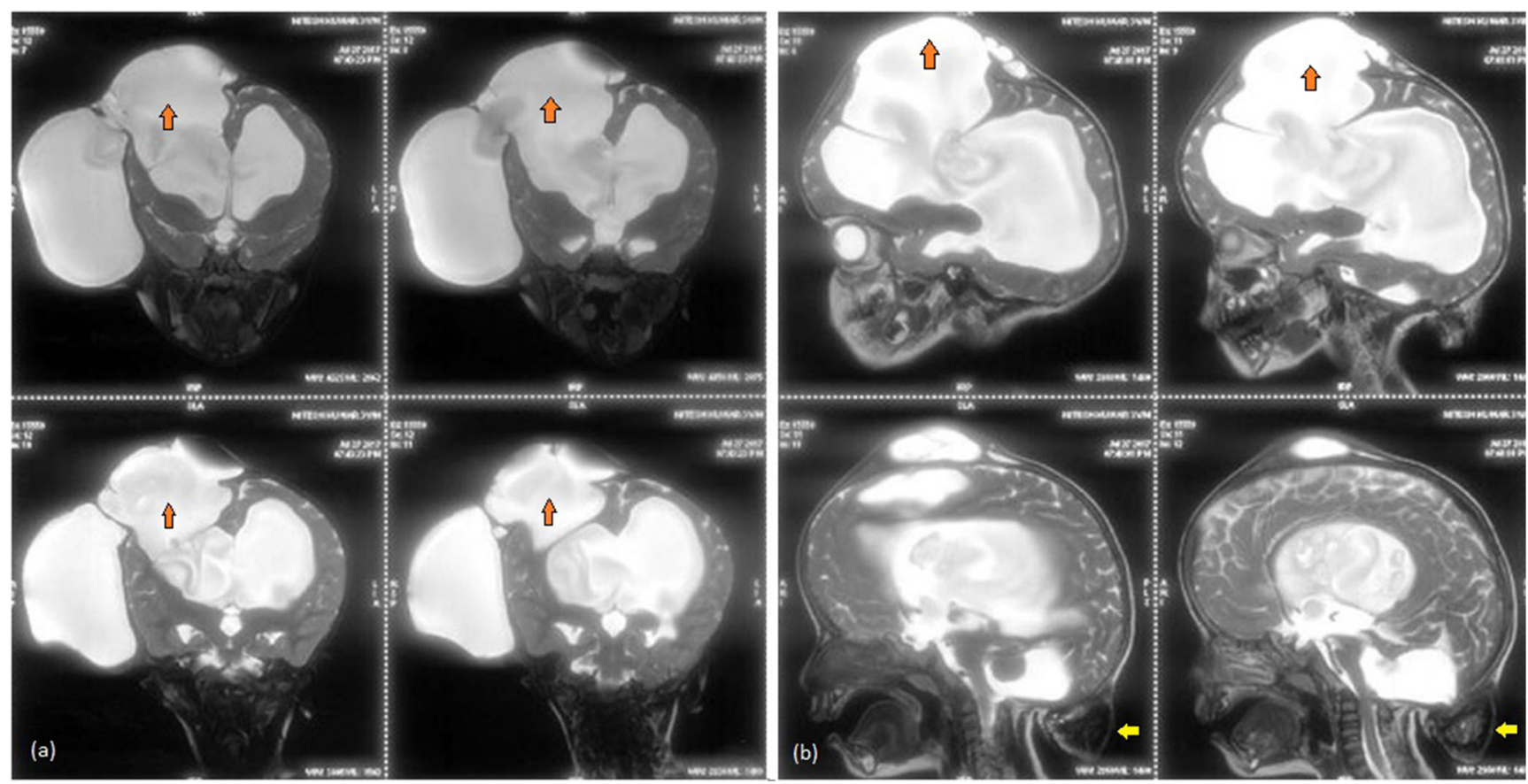

Fig. 3 MR T2 weighted images showing the dilated ventricles and their communication with the subgaleal collection (red arrow). Occipital encephalocele can also be seen (yellow arrow). a Coronal sections. b Sagittal sections

and internal and external rupture of hydrocephalus. The procedures for hydrocephalus are fairly simple and thus widely available these days. However, due to socio-cultural and economic factors as well as illiteracy, many cases present with neglected hydrocephalus in developing countries. In prenatal causes like aqueductal stenosis, the increasing hydrocephalus may result in loss of white matter and corpus callosal volume and lead to loss of septal leaflets or even ventricular rupture in intrauterine life itself [5]. With the delay in treatment, there is gradual thinning of the cerebral cortex and enlargement of the head especially in infants before sutural closure. If the pathologic process goes without intervention for long, it may lead to some odd complications like ventricular diverticulum [6] and even ventricular rupture. A few cases with internal rupture of enlarging ventricles into cisternal spaces or subdural space [7-9] and external rupture through the patent anterior fontanelle presenting with CSF leak with or without meningitis have already been reported in the literature (Table 1) [1-4, $10,11]$. Out of the 6 other cases of external rupture described in the literature, 2 were associated with lumbosacral myelomeningocele $[2,3]$ and in other 3 , congenital aqueductal stenosis $[1,4,10]$ was the cause of hydrocephalus while it was not mentioned in one. The present case is unique on two accounts. One, it is associated with occipital encephalocele and is only the second case to present with external rupture beyond the age of 1 year. The occipital swelling may not be a true encephalocele but a consequence of enlargement of the fourth ventricle mirroring the right frontal horn. Similarly, the occipital swelling may also be the result of a progressive meningocele associated with Dandy Walker malformation.
However, the MRI neither shows a direct communication between the ventricle and the contents of occipital swelling, nor does the swelling have CSF signal characteristics. Second, in this case, surprisingly, the ventricles have ruptured through the skull at the age of 2 years (after closure of anterior fontanelle) into the subgaleal space which later ruptured through the pressure sore on the skin. It is possible that the child had intrauterine infarction resulting in cortical mantle defect, giving a path of least resistance to the hydrocephalic ventricle to rupture. In the present case, we hypothesize that the persistent elevation of intracranial pressure after the closure of anterior fontanelle led to gradual resorption of the overlying bone at the site of maximum pressure with concomitant development of skin thinning due to difficulty in positioning of enlarged head. Complete resorption developed in a small region resulting in the rupture of hydrocephalus into subgaleal space. However, an unrecognized birth trauma leading to a localized right frontal bony defect may be the primary cause. It would result simultaneously in providing a preferred site for subgaleal rupture as well as premature closure of anterior fontanelle by the dissipation of intracranial pressure. The subgaleal collection further ruptured through the skin as the skin thinning progressed gradually and the ulcer deepened. This case brings into question the present understanding that external rupture occurs only through the open anterior fontanelle and after its closure only internal rupture may take place in the form of spontaneous ventriculostomy. In 2002, Ezechukwu et al. [10] reported a case with rupture of hydrocephalus at the age of 3 years; however, it is not 


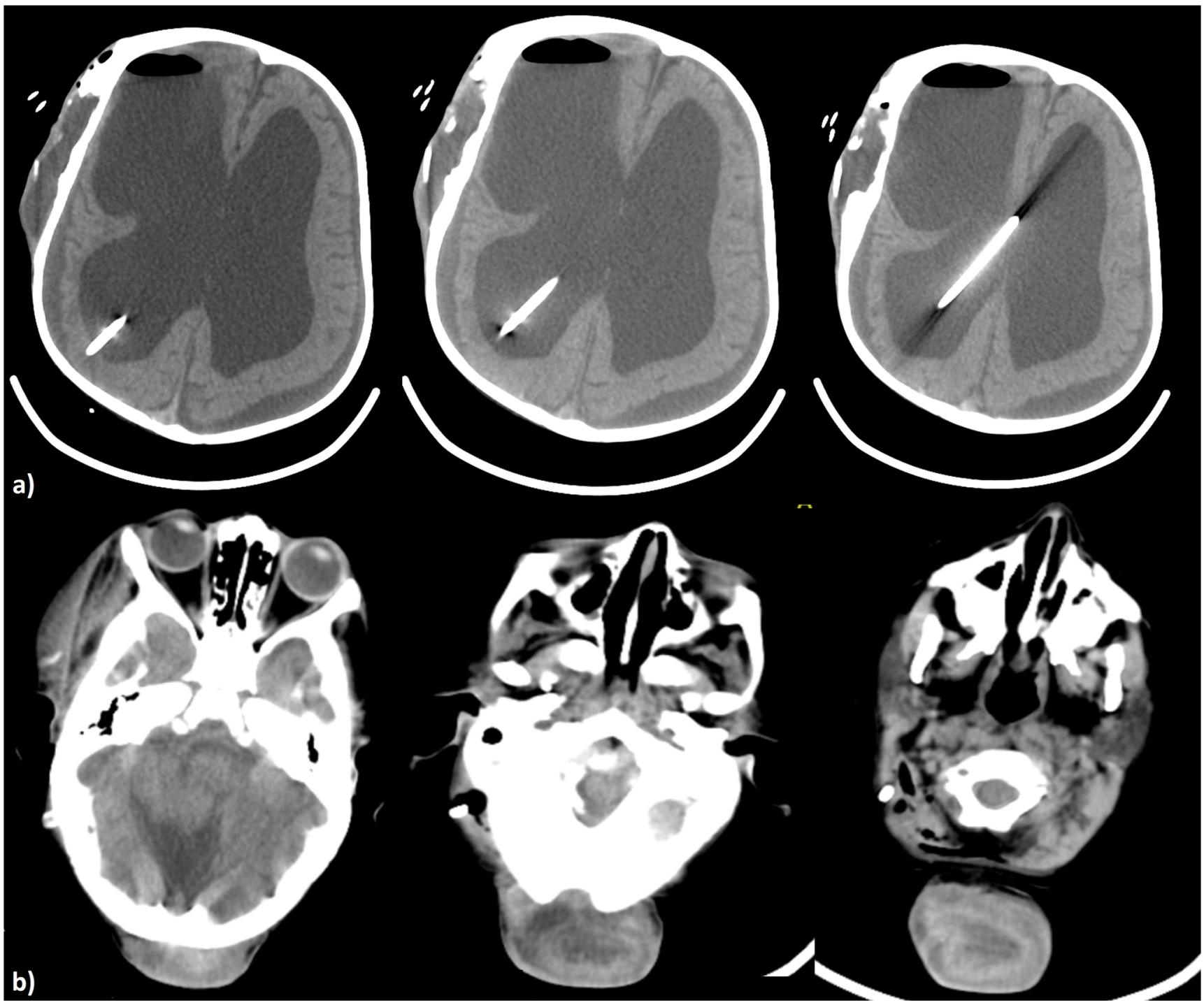

Fig. 4 Postoperative CT scan showing (a) the placement of right occipital medium pressure VP shunt, and (b) status of posterior fossa and encephalocele

clear from the report whether the fontanelle was open or closed and what was the exact course and site of rupture.

In all such cases of external rupture, close observation to detect and treat ventriculitis in a timely manner is required. Due to variability in the clinical presentation, the exact surgical treatment must be individualized. We planned a ventriculoperitoneal shunt thinking that it will result in decompression of the right lateral ventricle with resolution of subgaleal collection, which can later be followed by repair of the dural defect and cranioplasty. As observed by Tripathi

Table 1 Review of literature for cases with spontaneous external rupture of hydrocephalus

\begin{tabular}{llllll}
\hline Study & Age/Sex & Etiology & Site of rupture & Treatment & Outcome \\
\hline Ezechukwu et al., 2002 [10] & 3 years/female & Acqueductal stenosis & Not reported & None & Death \\
Moghtaderi et al., 2005 [1] & 3 months/male & Acqueductal stenosis & Anterior fontanelle & VP shunt and cranioplasty & Lost to follow up \\
Garg et al., 2013 [2] & 6 months/female & Chiari II malformation & Anterior fontanelle & & Death \\
Mishra et al., 2014 [3] & 8 months/female & Chiari II malformation & Anterior fontanelle & VP shunt & Not reported \\
Tripathi et al., 2015 [4] & 11 months/male & Acqueductal stenosis & Anterior fontanelle & VP shunt and cranioplasty \\
Oyemolade et al., 2019 [11] & 5 months/male & Not mentioned & Left occipital region & None & Death \\
Present case & 3 years/male & Occipital encephalocele & Right frontal bone & VP shunt & Under follow up \\
\hline
\end{tabular}


et al. [4], when combined with repair of the defects, decompression of the pathological ventricle results in the resolution of menigocoele with a fair prognosis.

\section{Conclusion}

Prolonged neglect of hydrocephalus may lead to bizarre complications like external rupture of ventricles through the skin. Unlike previously believed, this may occur even beyond infancy, after the closure of the anterior fontanelles, through skull defect caused by localized pressure induced resorption. All such cases with spontaneous external rupture require VP shunt to decompress the ventricles and repair of the dural and skull defects for prognosis to be good.

\section{Compliance with ethical standards}

Conflict of interest On behalf of all the contributors, the corresponding author states that there is no conflict of interest.

\section{Disclosure of funding None.}

Source of financial support None.

\section{References}

1. Moghtaderi A, Rahimi-Movaghar V, Safdari M (2005) Spontaneous brain rupture: a complication of untreated hydrocephalus. Clin Neurol Neurosurg 108:48-51. https://doi.org/10.1016/j. clineuro.2004.10.015
2. Garg K, Gurjar HK, Singh PK et al (2013) Spontaneous rupture of hydrocephalic head. Neurol India 61:556-558. https://doi.org/10. 4103/0028-3886.121955

3. Mishra SS, Mohanta I, Panigrahi S, Behera SK (2014) Spontaneous rupture of a neglected huge hydrocephalic head. J Pediatr Neurosci 9:203-204. https://doi.org/10.4103/1817-1745.139374

4. Tripathi M, Padwal AD, Shinde Pandit B, Shukla D, Bhat DI, Devi BI (2015) Unusual complication of neglected hydrocephalus: spontaneous rupture with growing skull fracture. Pediatr Neurosurg 50: 281-285. https://doi.org/10.1159/000438795

5. Kline-Fath BM, Arroyo MS, Calvo-Garcia MA, Horn PS, Thomas C (2018) Congenital aqueduct stenosis: progressive brain findings in utero to birth in the presence of severe hydrocephalus. Prenat Diagn 38:706-712. https://doi.org/10.1002/pd.5317

6. Garg K, Mahapatra AK (2012) A rare case of ventricular diverticulum in a child with occipital encephalocele. Pediatr Neurosurg 48: 26-29. https://doi.org/10.1159/000341726

7. Krishna Pillai M, Kariyattil R, Rubene L, Kumar R, Govindaraju V, Kochummen K (2018) Spontaneous ventriculostomy into the subdural space in a neonate with congenital hydrocephalus. Pediatr Neurosurg 53:188-192. https://doi.org/10.1159/000487087

8. Öğrenci A, Ekși MȘ, Koban O (2016) Spontaneous third ventriculostomy 8 years after diagnosis of obstructive hydrocephalus. Childs Nerv Syst 32:1727-1730. https://doi.org/10.1007/ s00381-016-3096-0

9. Timmers AAC, Bos EM, Dammers R (2019) A case of spontaneous third ventriculocisternostomy to the interpeduncular fossa. World Neurosurg 127:530-533. https://doi.org/10.1016/j.wneu. 2019.02.142

10. Ezechukwu CC, Egbuonu I, Chukwuka JO, Okoli CC (2002) Spontaneous rupture of a massive hydrocephalus resulting in head collapse. Niger J Clin Pract 5:148-149

11. Oyemolade TA, Balogun JA (2019) Spontaneous rupture: a rare complication of neglected hydrocephalus. Childs Nerv Syst 35: 2029-2031. https://doi.org/10.1007/s00381-019-04213-0

Publisher's note Springer Nature remains neutral with regard to jurisdictional claims in published maps and institutional affiliations. 